http://dx.doi.org/10.35381/racji.v6i11.1397

\title{
El Principio de inmediación en el desarrollo de audiencias virtuales en materia penal
}

The Principle of immediacy in the development of virtual hearings in criminal matters

Génesis Ivonne Macías-Sangoquiza

dq.genesisims78@unaindes.edu.ec

Universidad Regional Autónoma de los Andes, Quevedo

Ecuador

https://orcid.org/0000-0002-9387-8191

Luis Antonio Rivera-Velasco

uq.luisrivera@uniandes.edu.ec

Universidad Regional Autónoma de los Andes, Quevedo

Ecuador

https://orcid.org/0000-0003-2094-9566

Pamilys Milagros Moreno-Arvelo

uq.pamilysmoreno@uniandes.edu.ec

Universidad Regional Autónoma de Los Andes, Quevedo

Ecuador

https://orcid.org/0000-0001-8913-4352

Recepción: 31 de marzo 2021

Revisado: 30 de mayo 2021

Aprobación: 15 de junio 2021

Publicación: 01 de Julio 2021 


\title{
RESUMEN
}

A raíz de la pandemia mundial, el sistema procesal penal ecuatoriano, se ve en la obligación de maximizar la utilización de la metodología virtual, con la finalidad de que el sistema judicial no se detenga, generando una posible vulneración al principio de inmediación y por ende al debido proceso. Por todo ello la importancia del estudio de esta temática, cuyo objetivo general es realizar un análisis sobre la afectación y vulneración del principio de inmediación en los procedimientos virtuales en el ámbito penal del Ecuador. Se transitó bajo el enfoque cuantitativo, a través de la exploración, recolección y análisis crítico mediante una tipología documental, bibliográfica sobre el principio de inmediación. Con un diseño bibliográfico de tipo documental, que busca la reflexión y análisis crítico, y se construyan métodos relacionados de discernimiento del fenómeno y así evaluar o considerar nuevos contextos. Se concluye en la necesidad de reformas legales sobre virtualidad.

Descriptores: Derecho penal; telemática; legislación; juez; constitución. (Palabras tomadas de Tesauro UNESCO).

\begin{abstract}
As a result of the global pandemic, the Ecuadorian criminal procedure system is obliged to maximize the use of the virtual methodology, in order that the judicial system does not stop, generating a possible violation of the principle of immediacy and therefore due process. For all these reasons, the importance of studying this issue, the general objective of which is to carry out an analysis on the affectation and violation of the principle of immediacy in virtual proceedings in the criminal sphere of Ecuador. It was traveled under the quantitative approach, through exploration, collection and critical analysis through a documentary, bibliographic typology on the principle of immediacy. With a bibliographic design of a documentary type, which seeks reflection and critical analysis, and related methods of discernment of the phenomenon are built and thus evaluate or consider new contexts. It concludes in the need for legal reforms on virtuality.
\end{abstract}

Descriptors: Criminal law; telematics; legislation; judge; Constitution (words takenfrom UNESCO Thesaurus). 


\section{INTRODUCCIÓN}

El principio de inmediación, dentro del ordenamiento jurídico ecuatoriano se torna como uno de los principios fundamentales del debido proceso, y adquiere una importancia fundamental en el área penal, pues al decidir sobre los derechos de libertad o pena privativa de libertad de los procesados, es de vital importancia que el juzgador tenga pleno acceso directo a las partes, a las pruebas y a todo acto desarrollado cosa que mediante la virtualidad, con las limitaciones de contacto que esta conlleva, puede ponerse en peligro este principio de vital importancia para el debido proceso.

En este sentido la figura de inmediación como principio procesal, se encuentra enmarcada en el código orgánico integral penal en su artículo 5 numeral 17, en la cual se establece que el juez ponente del caso, está en la obligación de llevar a cabo la celebración de toda audiencia ante la presencia de los sujetos que son parte del proceso y este tendrá la obligación de estar presente en conjunto con las partes con la finalidad de desarrollar de la manera más transparente y equitativa los medios probatorios y de más actos correspondientes presentados por las partes y que conforman el procedimiento penal. (Código Orgánico Integral Penal, 2014).

De acuerdo a lo citado por Gallegos Rojas (2019, p121):

La inmediación a más de constituir uno de los principios fundamentales del sistema oral y de la actividad probatoria, implica: Un contacto directo entre el juez, las partes, terceros intervinientes y las pruebas, a fin de permitir la solución más adecuada y depuración más precisa de los hechos, lo que permite la observancia de los principios de la convicción racional del juez, de la inmediatez, de la publicidad, de la concentración y del incremento de los poderes instructores del juez (Martínez Lina y Díaz Laura, 2009)

Por otro lado, lo expresado por la Corte Nacional de Justicia de Ecuador (2020), las redes y tecnologías informáticas deben estar orientadas a ofrecer servicios inmediatos y veloces para aquellos que tengan la necesidad de acceder a la administración de justicia, para obtener resultados de mejor calidad, cumpliendo con los preceptos de una justicia de altas expectativas que abarque las necesidades de las partes procesales. Este tipo de sistemas deben servir como herramientas que permitan 
gestionar de una manera óptima lo procesos, para poder conseguir intercambios de información y comunicaciones ágiles, seguras y con un acceso sencillo y directo.

En este sentido, Arévalo Morales, Bautista Cruz y Gómez Pérez (2019) plantean que:

Ahora con la modalidad de las Audiencias Virtuales los acusados no necesitan ser trasladados fuera del recinto carcelario, haciendo el uso de internet, pantallas LED y cámaras en la cual la interacción desde el recinto penal y el despacho del juez se marca de una manera fluida como si fuese una audiencia presencial, pero que principios se debe tomar en cuenta que se pueden vulnerar. (p. 6)

Sin embargo, con base en lo que la Corte Nacional de Justicia establece en lo referente a los sistemas informáticos en la función judicial, cabe destacar, que Ecuador, afectado por la pandemia mundial se vio en la obligación de adelantar su adaptación a la metodología virtual, hecho por el cual se han desarrollado grandes falencia en el sistema jurídico de la actualidad, ocurriendo problemas de comunicación, tanto verbal como visual, en las audiencias penales, debido a que nuestro país todavía no cuenta con los elementos electrónicos eficaces para poder desarrollar la función judicial de manera óptima. Por ello a causa de la crisis mundial vivida en la actualidad, todos estos errores y fallos en la función judicial se han justificado, pero este problema es de vital importancia, pues se podría considerar que se están vulnerando los derechos de las personas en lo correspondiente al debido proceso y al principio de inmediación.

En contradicción, Palacio Riofrio (2016) plantea que:

El principio de inmediación exige la relación directa del juez con las partes y los elementos de prueba que él debe valorar para formar su convicción. Va estrechamente ligado con la oralidad del procedimiento. Cuando existe un intermediario, como ocurre en el proceso escrito, la convicción del juez se forma bajo influjos de comunicación preparada por un tercero, lo que puede traducirse en aumento del margen de error a la hora de emitir una sentencia. (p.12)

Otro principio a considerar y es necesario acotar es con la aplicación del principio de celeridad se logra evitar diligencias innecesarias, impedir acciones dilatorias e impugnación de las resoluciones, que obviamente repercute en la reducción del lapso 
y por consecuencias en la economía procesal. (Jarama Castillo, Vásquez Chávez, y Durán Ocampo, 2019, p.319)

Por todo ello la importancia del estudio de esta temática, cuyo objetivo general del presente trabajo es realizar un análisis sobre la afectación y vulneración del principio de inmediación en los procedimientos virtuales en el ámbito penal ecuatoriano.

\section{METODOLOGÍA}

El trabajo investigativo se transitó bajo el enfoque cuantitativo, a través de la exploración, recolección y análisis crítico mediante una tipología documental, bibliográfica sobre el principio de inmediación en el desarrollo de audiencias virtuales en materia penal en el Ecuador. Con un diseño bibliográfico de tipo documental, que busca la reflexión y análisis crítico, y se construyan métodos relacionados de discernimiento del fenómeno y así evaluar o considerar nuevos contextos. Al respecto, Palella y Martins (2012) la investigación documental se encuentra exclusivamente en la recolección de información en diversas fuentes. Por lo tanto, se consideraron varias leyes, normas, informes, tesis y trabajos científicos, con el objetivo de indagar y obtener la información relevante.

\section{RESULTADOS}

Los procesos judiciales se justifican en la medida que sirvan de medios para sustituir la justicia "de propia mano" con resguardo de los derechos y garantías fundamentales en tanto que áreas de libertad que deben ser respetadas por terceros y por el Estado $y$, al mismo tiempo, optimizadas por éste. Una de las grandes formas de mejorar el procedimiento judicial es a través de los avances tecnológicos conseguidos por la humanidad, destacando en este ámbito las tecnologías de comunicación e información, que representan una nueva era en la evolución de la humanidad, llevándonos a la era de la información.

En este sentido y desde este punto de vista, se observa de manera incoherente mantenerse de manera rígida en procedimientos creados en el siglo XX para satisfacer las necesidades de los procesos del siglo XXI, dando alusión a que se debe 
regresar en el tiempo para poder ejercer la justicia, cundo esta como tal debe avanzar y adaptarse a los tiempos cambiantes.

Por ello, se analizará la posibilidad real y jurídica de incorporar la videoconferencia en los procesos judiciales en respeto del principio de inmediación judicial, garantía prevista por el ordenamiento jurídico con el objeto de lograr una justicia más real, donde el juez tenga contacto directo con la fuente probatoria en lugar de acceder a ella mediante la lectura de un papel; o incluso para reducir costos y lapsos, así como también para incrementar la participación procesal, en especial de las personas alejadas de la sede judicial. (Amoni Rivero, 2013)

En enero de 2020, la Organización Mundial de la Salud (OMS) declaro la pandemia mundial COVID - 19, como una emergencia internacional. Ante la presencia de esta enfermedad en el territorio ecuatoriano en marzo del mismo año el ministerio de salud pública del Ecuador, mediante acuerdo ministerial No. 0012, declaró emergencia sanitaria en todo el territorio nacional por sesenta días el cual se extendió por treinta días más. El 16 de marzo de 2020, el presidente constitucional de la república a través de decreto ejecutivo No. 1710 establece estado de excepción por calamidad pública en todo el Estado ecuatoriano por sesenta días siendo este renovado por treinta días más el día 15 de mayo del 2020.

En cuanto a nivel jurisdiccional, la Corte Nacional de Justicia, mediante resolución, suspendió todo tipo de plazos o términos previstos pro ley para las judicaturas, suspendiendo de esta manera la atención al público de la función judicial mientras perduraran los efectos del estado de excepción debido a la emergencia sanitaria ocurrida por el COVID-19.

Para mayo del año 2020 el Consejo de la Judicatura mediante resolución decidió restablecer de manera parcial las actividades tanto de la corte nacional de justicia como de las cortes provinciales con la finalidad de que se despacharan todos los procesos que se encontraban en tramitación tanto en sistema oral como en el escrito. En la misma resolución antes mencionada del consejo de la judicatura, se dispuso que, en base a sus atribuciones la Corte Nacional de Justicia debía emitir una guía para el desarrollo de audiencias virtuales y que el Consejo de la Judicatura determinaría las especificaciones técnicas a observar por parte de las y los 
juzgadores, de forma que se "garantice el normal desenvolvimiento de las mismas". (Corte Nacional de Justicia, 2021)

El principio procesal de inmediación penal, implica la necesidad del desarrollo de una relación del juzgador tanto con las partes procesales como con todos los elementos probatorios que este debe valorar con la finalidad de formar un criterio de convicción. Cuando en la relación directa entre partes y juzgador existe un intermediario, tal como ocurre en el proceso escrito, los elementos que deben desarrollar la convicción al juzgador se forman en base a comunicaciones preparadas por un tercero, lo que conlleva a una ampliación del margen de error que puede tener un juzgador a la hora de emitir una resolución a un caso concreto. (Palacio Riofrio, 2016)

Por lo tanto, el principio de inmediación penal persigue que el juzgador pueda adquirir una percepción directa de los hechos que han sucedido de manera directa, sin la necesidad de existencia de intermediarios y que estos hechos que en sentencia se estimen hayan sido correctamente probados. Por lo tanto, podemos decir que este principio busca que el juzgador perciba mediante sus propios sentidos la realidad de los hechos controvertidos, pues son las partes procesales las que deberán desarrollar afirmaciones acerca de los hechos que se han suscitado en base a sus capacidades de interpretación memoria, percepción y narración de los sucesos que componen los hechos delictuales.

\section{Cuadro 1.}

Principio de inmediación en la audiencia telemática.

\begin{tabular}{|l|l|}
\hline \multicolumn{1}{|c|}{ Ordenamiento jurídico } & \multicolumn{1}{|c|}{ Artículos } \\
\hline Código Orgánico Integral Penal (2014) & $\begin{array}{l}\text { Artículo 5.-Numeral 17 Inmediación: La o el } \\
\text { juzgador celebrará las audiencias en } \\
\text { conjunto con los sujetos procesales y } \\
\text { deberá estar presente con las partes para } \\
\text { la evacuación de los medios de prueba y } \\
\text { demás actos procesales que estructuran de } \\
\text { manera fundamental el proceso penal. } \\
\text { Artículo 502.- Numeral 2 La o el juzgador } \\
\text { podrá recibir como prueba anticipada los } \\
\text { testimonios de las personas gravemente } \\
\text { enfermas, de las físicamente } \\
\text { imposibilitadas, de quienes van a salir del }\end{array}$ \\
\hline
\end{tabular}




\begin{tabular}{|c|c|}
\hline & $\begin{array}{l}\text { país, de las víctimas o testigos protegidos, } \\
\text { informantes, agentes encubiertos y de } \\
\text { todas aquellas que demuestren que no } \\
\text { pueden comparecer a la audiencia de juicio. } \\
\text { En el caso de audiencia fallida, y en los que } \\
\text { se demuestre la imposibilidad de los } \\
\text { testigos de comparecer a un nuevo } \\
\text { señalamiento, el tribunal, podrá receptar el } \\
\text { testimonio anticipado bajo los principios de } \\
\text { inmediación y contradicción. } \\
\text { Articulo } 610 .- \text { En el juicio regirán, } \\
\text { especialmente los principios de oralidad, } \\
\text { publicidad, inmediación y contradicción en } \\
\text { la actuación probatoria. Asimismo, en su } \\
\text { desarrollo se observarán los principios de } \\
\text { continuidad del juzgamiento, concentración } \\
\text { de los actos del juicio, identidad física de la } \\
\text { o el juzgador y presencia obligatoria de la } \\
\text { persona procesada y de la o el defensor } \\
\text { público o privado, con las salvedades del } \\
\text { juzgamiento en ausencia previstas en la } \\
\text { Constitución. }\end{array}$ \\
\hline \begin{tabular}{|llll} 
Constitución de la & República & Del \\
Ecuador(2008)
\end{tabular} & $\begin{array}{l}\text { Artículo. 75.-Toda persona tiene derecho al } \\
\text { acceso gratuito a la justicia y a la tutela } \\
\text { efectiva, imparcial y expedita de sus } \\
\text { derechos e intereses, con sujeción a los } \\
\text { principios de inmediación y celeridad; en } \\
\text { ningún caso quedará en indefensión. El } \\
\text { incumplimiento de las resoluciones } \\
\text { judiciales será sancionado por la ley. }\end{array}$ \\
\hline
\end{tabular}

Elaboración: Los autores.

La importancia del Principio de Inmediación radica en la idea de que la relación física y directa del juzgador con las partes y con las pruebas rendidas es la fórmula más óptima, o el instrumento más útil para lograr el convencimiento personal de mejor calidad del juzgador, ya que la prueba debe ser apreciada conforme a las reglas de la Sana Crítica. Como se puede evidenciar la valoración de la prueba es una actividad compleja y en este punto la inmediación es condición necesaria para tales fines, de allí entonces, su importancia en el desarrollo del juicio oral. 


\section{DISCUSIÓN}

Como punto de partida para discusión de nuestra temática se encuentra la respuesta a la pregunta: ¿se satisface el estándar del principio de inmediación con la presencia virtual del Tribunal? ¿O necesitamos su presencia física?

La ley y la jurisprudencia ecuatoriana establecen que el principio de inmediación exige de manera obligatoria la relación directa del juzgador con todas las partes activas del proceso, así como su implicación con todos los elementos de prueba que debe valorar para desarrollar un criterio propio y personal, para establecer su convicción de los hechos que se hayan suscitado. Cuando surge la existencia de un factor externo dentro del procedimiento, se refiere en este caso al desarrollo de audiencias a través de medios telemáticos, la convicción del jugador puede verse afectado por diversos factores, ya sea conectividad, intromisión de terceros en los actos probatorios, al no saber cómo o con quien se encuentran las partes, o si estas están siendo incitadas de una u otra forma a declarar de manera a conveniencia, se desarrolla la problemática de la vulneración directa del principio de inmediación en el desarrollo de audiencias virtuales.

Las video conferencias dentro del sistema procesal es un tipo de sistema de comunicación que son de auténtica utilidad, a la hora de desarrollar diligencias tales como desarrollo de auxilios judiciales tanto de forma nacional como internacional, conformación de comisiones rogatorias, aplicación de ruedas de reconocimiento, desarrollo y aplicación de entrevistas realizadas por juzgadores hacia reclusos, captación de declaraciones, interrogatorios o entrevistas a menores de edad en unidades judiciales.

Así mismo, las audiencias virtuales, pueden servir de auxiliar del procedimiento judicial, pero si esta herramienta se vuelve el eje principal del desarrollo de la práctica judicial, surgen grandes problemáticas en diferentes ámbitos, como la conectividad, la presencia de las partes, la relación directa del juzgador con las partes, o el desconocimiento de la utilización de este tipo de sistemas y plataformas por parte de juzgadores y profesionales del derecho, lo cual dificulta el correcto proceder de las audiencias, hecho por el cual surgen las distintas críticas hacia esta modalidad, y por consiguiente la valoración sobre posibles vulneraciones a los principios procesales, 
los cuales no contemplan en la ley o en su costumbre la virtualidad, hecho que debe evolucionar. puesto que la tecnología y la información son el futuro de la humanidad, y el derecho no será una excepción a la adaptación a la tecnología, es por ello que se debe prever dentro de la ley la evolución y la tecnología en los preceptos que rigen el ordenamiento jurídico ecuatoriano.

\section{CONCLUSIONES}

Desde la fundamentación teórica expuesta se logra concluir que el principio de inmediación establece según la ley y la doctrina la necesidad de que la o el juzgador tenga un contacto directo y físico con las partes del proceso y con la actividad probatoria, con la finalidad de que este obtenga los elementos de convicción necesarios para poder desarrollar un criterio adecuado con la cual resolver la causa puesta en su conocimiento, sin tomar en cuenta una aplicación de este principio mediante vías telemáticas, hecho por el cual se genera la problemática de una posible vulneración al principio de inmediación a través del desarrollo de audiencias telemáticas. Para finalizar se establece la necesidad de reformas legales completas al sistema procesal, en la cual se prevea a la virtualidad no solo como un auxiliar de la función judicial, sino como representante del eje principal del desarrollo de la función judicial en caso de ser necesario.

\section{FINANCIAMIENTO}

No monetario.

\section{AGRADECIMIENTO}

A la Universidad Regional Autónoma de los Andes, Quevedo, por motivar el desarrollo de la Investigación. 


\section{REFERENCIAS CONSULTADAS}

Amoni Reverón, G. (2013). El uso de la videoconferencia en cumplimiento del principio de inmediación procesal. [The use of videoconferencing in compliance with the principle of procedural immediacy] Revista IUS, 7(31), 67-85. Recuperado de: https://n9.cl/drn4n

Arévalo Morales, Y., Bautista Cruz, H. y Gómez Pérez, V. (2019) La flexibilización del principio de inmediación con la realización de audiencias virtuales en el proceso penal. [The relaxation of the principle of immediacy with the holding of virtual hearings in criminal proceedings]. Trabajo de Grado para obtener el título de Licenciado $(A)$ en Ciencias Jurídicas. Universidad de El Salvador. http://ri.ues.edu.sv/id/eprint/19766/

Asamblea Nacional Constituyente de la República del Ecuador, (2008). Constitución de la República del Ecuador. [ Montecristi. Registro Oficial 449 de 20-oct-2008. Recuperado de https://n9.cl/sia

Asamblea Nacional (2014). Código Orgánico Integral Penal. [Comprehensive Criminal Code]. Registro Oficial № 180. Recuperado de https://url2.cl/53c6h

Corte Nacional de Justicia (2021) Protocolo para la realización de audiencias telemáticas en la Corte Nacional de Justicia. [Protocol for conducting telematic hearings in the National Court of Justice].Recuperado de: https://n9.cl/zzeqd

Gallegos Rojas, R. (2019) El principio de inmediación y la actividad probatoria en la normativa procesal ecuatoriana. [ The principle of immediacy and probative activity in the Ecuadorian procedural law]. INNOVA Research Journal, 4(2), 120-131. DOI: https://doi.org/10.33890/innova.v4.n2.2019.978

Jarama Castillo, Z., Vásquez Chávez, J., y Durán Ocampo, A. (2019). El principio de celeridad en el código orgánico general de procesos, consecuencias en la audiencia. [ The principle of speed in the general organic code of processes, consequences in the audience]. Revista Universidad y Sociedad, 11(1), 314323. Recuperado de: https://n9.cl/dpzc3

Organización Mundial de la Salud. (2013). COVID-19: cronología de la actuación de la OMS. Recuperado de: https://n9.cl/yx6h6

Palacio Riofrio, M. (2016) Vulneración del principio de inmediación establecido en el Art. 610 del Código Orgánico Integral Penal, por la aplicación de la audiencia telemática en la etapa de juicio. [Violation of the principle of immediacy established in Article 610 of the Organic Integral Criminal Code, by the application of the telematic hearing in the trial stage]. Tesis previa a la obtención del Título de Abogada. Universidad Nacional de Loja. Ecuador. Recuperado de: https://n9.cl/hgijg 
Palella, S. y Martins, F. (2012). Metodología de la Investigación Cuantitativa. [Quantitative Research Methodology]. Fondo Editorial de la Universidad Pedagógica Experimental Libertador. Caracas. Venezuela

Presidencia de la República de Ecuador. (2020). Decreto presidencial № 1017. [Presidential Decree No. 1017]. Recuperdo de: https://n9.cl/f3kkd 